\title{
CARACTERIZACIÓN BIOMÉTRICA DE LAS MADERAS \\ DE Picea obovata Ledeb., Chamaecyparis lawsoniana (A. Murray) \\ Parlatore y Pinus strobus L. CRECIDAS EN EL SECTOR LOS \\ GUINDOS DE LA RESERVA FORESTAL DE MALLECO \\ (CHILE).
}

ROBERTO MELO S ${ }^{\left({ }^{(*)}\right.}$ y LUTS MORAGA V ${ }^{(*)}$

(*) Ingeniero Civil Químico, Laboratorio de Productos Forestales, Universidad de Concepción.

${ }^{* *}$ ) Ingeniero Forestal, Universidad de Concepción.

\section{RESUMEN}

Peso especifico, longitud de traqueida y peso unitario de fibra (coarseness) fueron medidos en árboles de 60 años de las especies Picea obovata Ledeb., Chamaecyparis lawsoniana (A. Murray) Parlatore y Pinus strobus L., crecidas en la Reserva Forestal de Malleco, Chile.

Los promedios de longitud de traqueida para las tres especies se encuentran alrededor de $2,5 \mathrm{~mm}$, siendo inferiores a los informados por la literatura. El margen de peso especifico a volumen saturado para las especies fluctuó entre 0.30 y 0.36. Para Picea obovata Ledeb. $y$ Pinus strobus $L$. los valores de peso unitario de fibra se situaron entre 17 y $18 \mathrm{dg}$, en tanto para Chamaecyparis lawsoniana (A. Murray) Parlatore no sobrepasó el valor de 12 dg.

Se informa también sobre la variación de estas propiedades tanto en la altura del árbol como desde médula a corteza.

Palabras Claves: Coniferas, Peso especifico, Longitud de traqueida, Coarseness.

\section{ABSTRACT}

Specific gravity, fiber length and coarseness have been measured from Picea obovata Ledeb., Chamaecyparis lawsoniana (A. Murray) Parlatore and Pinus strobus L., 60 years old. grown in the Malleco Forest Reserve, Chile.

Average values of fiber length are around $2,5 \mathrm{~mm}$ for the three species, wich are below those reported by literature. Specific gravity ranged within 0,30 and 0,36 for these species, whereas coarseness was 17-18 dg for Picea obovata Ledeh. and Pinus strobus L., and less than $12 \mathrm{dg}$ for Chamaecyparis lawsoniana (A. Murray) Parlatore.

Variation of these properties with tree height and radial in the trunk are also reported. Keywords: Conifers, Specific gravity, Fiher length, Coarseness. 


\section{INTRODUCCIÓN}

De los criterios usados para determinar la calidad de la madera, el peso específico es el más importante puesto que se relaciona de manera directa con la resistencia de la madera y con los rendimientos de pulpa para la industria papelera. siendo a su vez. el principal indicador de la cantidad de fibra seca de la madera (Daniel et al., 1982; González y Molina, 1989; Timell (1986), citado por Vargas, 1991). De acuerdo a L. P. F. (1985), la densidad es la característica física más significativa para determinar la calidad de la madera y la pulpa, pues engloba en una sóla variable los factores como edad, posición en el fuste, velocidad de crecimiento, tratamientos silvícolas, calidad del sitio y herencia. El fenómeno anterior ha motivado a numerosos investigadores al estudio del comportamiento de esta característica de los árboles maderables, llegándose a determinar, en primera instancia, que el peso específico varía en función de muchos factores que condicionan el desarrollo de las masas boscosas (Gysling, 1982), siendo, a su vez, según van Buijtenen (1964) citado por Zobel y Talbert (1988), no una característica simple de la madera, sino una combinación de características, cada una de las cuales posee un sólido patrón hereditario propio. Combinados, finaliza la cita. determinan lo que se conoce como peso específico.

El largo de traqueida, por otro lado. es considerada una de las cualidades más importantes de la madera. especialmente por su efecto en las resistencias y características superficiales del papel, así como en la resistencia al rasgado y al doblez (Casey. 1960: Daniel et al., 1982). Es así como Dadswell y Nicholl (1959) citados por Delmastro et al. (1980), concluyen que el largo de traqueida, la densidad y la contracción longitudinal son las tres determinaciones que entregan prácticamente toda la información requerida de una madera. Por la relativa facilidad para determinarla, y por ser la dimensión que proporciona la información más útil, es incluida en casi todos los estudios técnicos relacionados con la pulpa y el papel (Einspahr, 1964).

Finalmente. en la caracterización de la morfologia de la fibra. un importante factor ha de ser tomado en cuenta: tal es el peso por unidad de longitud o "coarseness de fibra" (Britt. 1966). Esta propiedad de las células conjuga la relación longitud/diámetro y la morfologia de la sección transversal (L. P. F., 1985). Casey (1960), al estudiar el concepto. lo define como una razón entre longitud y diámetro de la fibra. Incluye este factor, agrega, los efectos del grosor de la fibra, el tamaño del canal central o lumen y la densidad del material sólido que la compone. Por otra parte. Martinez y Pacheco (1990), señalan que el indice de coarseness representa el espesor de pared de la fibra y, de acuerdo a Delmastro et al. (1980), al igual que otras dimensiones celulares, expresa en términos morfológicos la cantidad y distribución de la sustancia celular en el volumen de madera. influyendo en la mayoría de sus propiedades y, por ende, en la calidad y posibilidades de uso de este material, teniendo especial relevancia en la madera destinada a la producción de pulpa y papel. En este sentido. Casey (1960) 
apunta: "puesto que los fabricantes de papel manejan más bien la pulpa en dimensiones de peso, el registro de pesos promedios de fibra por unidad de longitud de ésta, parece ser mucho más razonable que un simple promedio aritmético de tal longitud".

El propósito de este trabajo es caracterizar biométricamente la madera de las especies Picea obovata Ledeb., Chamaecyparis lawsoniana (A. Murray) Parlatore y Pinus strobus $\mathbf{L}$., de modo de generar información que sea potencialmente útil para propender a la diversificación de las plantaciones forestales en Chile y para apoyar las decisiones que se tomen en el manejo del recurso que se crea y en la utilización del existente.

Se han planteado como objetivos específicos la determinación de tendencias y valores promedios de las propiedades peso específico a volumen saturado, longitud de traqueida y coarseness en la madera de las especies mencionadas.

\section{MATERIAL Y MÉTODO}

Descripción del Área de Estudio (CONAF, 1975).

El área de estudio corresponde al sector Los Guindos de la Reserva Forestal de Malleco. ubicada en la comuna de Collipulli. IX Región de la Araucanía.

El clima se clasifica como templado cálido con influencia mediterránea. Las precipitaciones sólidas son escasas. concentrándose las lluvias en invierno (superiores a $2400 \mathrm{~mm}$ ), las que están muy por sobre las estivales $(330 \mathrm{~mm})$. La temperatura promedio anual fluctúa entre $13^{\circ} \mathrm{C}$ y $14^{\circ} \mathrm{C}$.

Los suelos corresponden a depositaciones andesitico - basálticas provenientes de erupciones volcánicas. Conocidos como trumaos de altura. poseen una gran proporción de arcilla alófana tipo amorfa, lo que explica una estructura muy porosa y relativamente estable. pero de consistencia muy suelta en seco. La fertilidad de estos trumaos de altura es relativamente alta debido a su gran contenido en minerales fácilmente meteorizables, la poca lixiviación. la mediana capacidad de cambio y su buena estructura y alta estabilidad frente a la erosión cuando se conserva una cobertura permanente de vegetación. La caracterización de los terrenos de la Reserva permite, en su totalidad. clasificarlos en las clases de uso VII y VIII.

\section{Descripción de los Rodales en Estudio.}

En el Cuadro $\mathrm{N}^{\circ} 1$ se incluye información dasométrica básica que entrega para las especies en estudio la edad en años. el DAP medio en centímetros. la altura media en metros. el número de árboles totales y el área basal total en metros cuadrados. 
Cabe hacer notar que el rodal de la especie Chamaecyparis lawsoniana (A. Murray) Parlatore (Ciprés de Lawson) estaba constituido por ejemplares ordenados a lo largo de un camino predial, conformando de este modo una hilera o avenida. Los rodales de las especies Picea obovata Ledeb. (Picea Siberiana) y Pinus strobus L. (Pino Blanco del Este), en tanto, correspondian a la ordenación de la plantación experimental establecida en la década de 1930.

\section{Cuadro $\mathrm{N}^{\circ} 1$}

INFORMACIÓN DASOMÉTRICA BÁSICA

\begin{tabular}{|l|c|c|c|c|c|}
\hline \multicolumn{1}{|c|}{$\begin{array}{c}\text { Especie } \\
(-)\end{array}$} & Edad & DAP medio & Altura media & $\begin{array}{c}\text { Árboles } \\
\text { totales } \\
\left(\mathrm{N}^{\circ}\right)\end{array}$ & $\begin{array}{c}\text { Área basal } \\
\text { total } \\
\left(\mathrm{m}^{2}\right)\end{array}$ \\
\hline Picea obovata & $($ años $)$ & $(\mathrm{cm})$ & $(\mathrm{m})$ & 81 & 10.3 \\
Chamaecyparis lawsoniana & \multirow{2}{*}{60} & 38.1 & 24.4 & 49 & 11.3 \\
Pinus strobus & & 58.5 & 22.2 & 24.4 & 7.0 \\
\hline
\end{tabular}

Fuente: Bases Licitación "Estudios Básicos sobre volumetria. crecimiento y taxonomía en rodales de coniferas exóticas en Sector Los Guindos. Reserva Forestal de Malleco". (1991). CONAF. Dirección Regional IX Región.

Tipo de muestreo y selección de los árboles.

Dadas las características de los rodales (escaso número de individuos y, en algunos casos. coexistencia de especies diferentes). se optó por realizar líneas de muestreo de 50 $\mathrm{m}$ de largo para la especie Chamaecyparis lawsoniana (A. Murray) Parlatore y parcelas de $500 \mathrm{~m}^{2}$ para Picea obovata Ledeb. y Pinus strobus L., determinando el DAP de todos los individuos, para posteriormente confeccionar Tablas de Rodal desde las cuales se determinó el DAP promedio en base al que se seleccionaron los árboles respectivos. De este modo se seleccionaron un mínimo de tres árboles por especie. correspondiendo uno a la clase del DAP medio. otro a la clase superior y el tercero a la clase inferior a dicho diámetro.

Los valores correspondientes al DAP de los árboles muestreados de cada especie se exponen en el Cuadro $\mathrm{N}^{\mathrm{N}} 2$. 


\section{Cuadro $\mathrm{N}^{\circ} 2$ \\ INFORMACIÓN DASOMÉTRICA DE LOS ÁRBOLES MUESTREADOS}

\begin{tabular}{|c|c|c|c|}
\hline \multirow{2}{*}{$\begin{array}{c}\text { Especie } \\
(-)\end{array}$} & \multicolumn{3}{|c|}{$\operatorname{DAP}(\mathrm{cm})$} \\
\hline & Árbol $N^{2} 1$ & Árbol $N^{2} 2$ & Árbol N $N^{2} 3$ \\
\hline Picea obovata & 35,0 & 25,0 & 46,0 \\
\hline Chamaecyparis lawsoniana & 47,0 & 57.0 & 64,0 \\
\hline Pinus strobus & 78,0 & 64,0 & 70,0 \\
\hline
\end{tabular}

\section{Obtención de las Muestras para Experimentación.}

Las muestras para la determinación de las propiedades biométricas correspondieron a material proveniente de rodelas tomadas a diferentes alturas, en los árboles seleccionados. Estas, de aproximadamente $5 \mathrm{~cm}$ de espesor, se identificaron convenientemente de acuerdo a un código que denotaba la especie y la altura a la que correspondia.

En los casos de presencia de anomalías, como nudos, pudriciones o quebraduras en el fuste, se procedió a tomar la rodela en la zona contigua al área anómala de modo que la madera muestreada no tuviese defectos que alteraran las propiedades por evaluar.

Para la determinación del comportamiento intraincremental en altura y edad del peso especifico a volumen saturado, longitud de traqueida y coarseness de madera, se retiraron cuatro cuñas opuestas por rodela, las que se dividieron a su vez en dos sectores de un centimetro de arco, constituyendo finalmente dos muestras de experimentación de cuatro sectores cada una. De tales muestras se obtuvieron subsectores de diez anillos de crecimiento, los que agregados según incrementos, se fraccionaron en astillas de un $\mathrm{cm}$ de longitud y un mm de espesor, constituyendo el material de trabajo para los análisis intraincrementales.

Para cada una de las especies, el árbol de DAP medio proveyó la mayor proporción de material. Es así como en la determinación del peso especifico se generaron cuñas desde todas las rodelas de este ejemplar, en tanto que los dos restantes árboles generaron cuñas desde sólo algunas de sus diferentes rodelas. En este caso, el criterio de selección de las cuñas fue la disponibilidad y calidad del material y la coincidencia en altura de las rodelas elegidas. Por otro lado. en la determinación de la longitud de traqueidas y coarseness de madera se seleccionaron únicamente tres rodelas del árbol de DAP medio para generar el material de experimentación (inferior, media y superior). 


\section{Determinación del Peso Específico de la Madera.}

El peso específico de la madera se determinó de acuerdo al método de desplazamiento de agua según volumen de la pieza, conocido como "Método de Arquímedes". Este método, que permite relacionar peso anhidro con volumen saturado o verde, es ampliamente citado por la literatura como procedimiento de determinación del peso específico. El estado anhidro se obtuvo colocando las probetas en una estufa a $103^{\circ} \mathrm{C}$ (con una variación de $2^{\circ} \mathrm{C}$ ) durante 48 horas o hasta peso constante: y el volumen saturado. tras someterlas a inmersión hasta su precipitación total en los depósitos destinados para tal efecto, midiendo el incremento en peso que experimenta un vaso de precipitado conteniendo agua corriente cuando se introduce en él la probeta soportada por un fino sostén punzante.

Las mediciones fueron hechas con una balanza analítica - en caso de muestras pequeñas - o de mayor capacidad, en el caso de piezas grandes. La probeta saturada, antes de ser pesada. fue suspendida sobre el agua desde el brazo de la balanza o desde un tripode acondicionado para ello. La balanza, asi como los dispositivos accesorios, se calibraron y pesaron antes de la inmersión de las piezas. siendo la precisión exigida en los ensayos de $0.01 \mathrm{~g}$. con tres repeticiones para cada determinación a partir de las cuales se obtuvo un valor promedio.

El concepto de peso especifico aquí adoptado. es el de una relación de dimensiones expresado en función del volumen base: "Peso especifico a volumen saturado".

La bondad y aplicación del método se debe a que, siendo la densidad del agua virtualmente unitaria a temperatura ambiente. la diferencia de los registros de la balanza antes y después de sumergir la probeta. corresponde al volumen de agua desplazado. esto es. al volumen de la probeta.

Determinación de la Longitud de Traqueidas y del Coarseness de Madera.

La metodología seguida. se basó en los procedimientos descritos en "Tappi Test Methods". Volumen $\mathrm{N}^{\circ} 1$ (1989): publicados por la "Technical Association of the Pulp and Paper Industry (TAPPI)".

Las Normas empleadas en este estudio fueron:

- Norma TAPPI T $232 \mathrm{~cm}-85$ "Fiber length of Pulp by projection".

- Norma TAPPI T $234 \mathrm{~cm}-84$ "Coarseness of Pulp fibers". 


\section{Análisis de los Datos.}

Para cada una de las especies, los resultados hallados fueron analizados para determinar los valores promedios y conocer las tendencias de las propiedades estudiadas.

El proceso de cálculo de promedios y tendencias consideró la influencia del volumen de las piezas, por lo que debió ponderarse los valores según dicha variable. En otras palabras, se determinó la influencia relativa que cada sector intraincremental ejercía sobre el total de la rodela y, por extensión, sobre el volumen total del árbol y, por ende. sobre cada una de las propiedades medidas.

\section{RESULTADOS}

\section{Valores Promedios Ponderados.}

El Cuadro $\mathrm{N}^{\circ} 3$ muestra los valores promedios determinados en este estudio para cada una de las tres propiedades biométricas determinadas en las maderas evaluadas. En el caso particular de las cualidades peso especifico a volumen saturado y longitud de traqueida, se detectan valores que están por bajo aquellos informados por la literatura para las mismas especies, si bien ambas se encuentran dentro del margen definido para coniferas. En relación a los valores de coarseness de madera. aunque no se informan promedios generales para gimnospermas. las especies estudiadas presentan promedios ponderados próximos a los informados para otras especies coníferas (aunque de edades diferentes a los ejemplares evaluados en esta investigación).

\section{Cuadro $\mathrm{N}^{\circ} 3$}

PROMEDIOS PONDERADOS SEGÚN ATRIBUTO Y ESPECIE

\begin{tabular}{|l|c|c|c|}
\hline \multicolumn{1}{|c|}{$\begin{array}{c}\text { Especie } \\
(-)\end{array}$} & $\begin{array}{c}\text { Peso especifico } \\
(\mathrm{g} / \mathrm{cm} 3)\end{array}$ & $\begin{array}{c}\text { Longitud de traqueida } \\
(\mathrm{mm})\end{array}$ & $\begin{array}{c}\text { Coarseness } \\
(\mathrm{dg})\end{array}$ \\
\hline Picea obovata & 0.363 & 2,66 & 17.64 \\
Chamaecyparis lawsoniana & 0.360 & 2.41 & 12,00 \\
Pinus strohus & 0.297 & 2.64 & 17.99 \\
\hline
\end{tabular}

\section{Tendencias del Peso Específico en Relación a la Altura del Árbol.}

En el Cuadro $\mathrm{N}^{\circ} 4$ se resumen los resultados obtenidos en las determinaciones de peso específico promedio ponderado a diferentes alturas para las tres especies bajo estudio. La discusión de los mismos se presenta en forma individual según el orden establecido en el Cuadro mencionado. 


\section{Cuadro $N^{\circ} 4$}

PESO ESPECIFICO PROMEDIO PONDERADO A VOLUMEN SATURADO EN RELACIÓN A LA ALTURA DEL ÁRBOL SEGÚN ESPECTE

\begin{tabular}{|c|c|c|c|}
\hline \multirow{2}{*}{$\begin{array}{c}\text { Altura } \\
(\mathrm{m})\end{array}$} & \multicolumn{3}{|c|}{ Peso Especifico (g/cm3) } \\
\cline { 2 - 4 } & Picea obovata & Chamaecyparis lawsoniana & Pimus strobus \\
\hline 0,25 & 0,400 & 0,372 & 0,336 \\
3,95 & 0,354 & 0.345 & 0,283 \\
5,20 & - & 0,378 & 0,289 \\
6,45 & 0,369 & 0,345 & 0,270 \\
10,15 & 0,369 & 0,353 & 0,296 \\
10,85 & - & 0,363 & - \\
11,40 & - & 0,343 & 0,271 \\
12.65 & 0,358 & 0.333 & 0,282 \\
16,35 & 0,346 & 0.353 & 0,298 \\
17.05 & - & 0,392 & - \\
20,05 & - & - & 0,301 \\
23,75 & - & - & 0,325 \\
\hline
\end{tabular}

La tendencia seguida por los valores promedios ponderados en la madera de Picea obovata Ledeb. refleja una disminución gradual del peso específico con la altura. Tal relación se ilustra a través de la Figura $\mathrm{N}^{\circ}$ 1, la que presenta para Picea Siberiana la tendencia del peso especifico en sectores intraincrementales equivalentes según sucesivas alturas en el árbol.

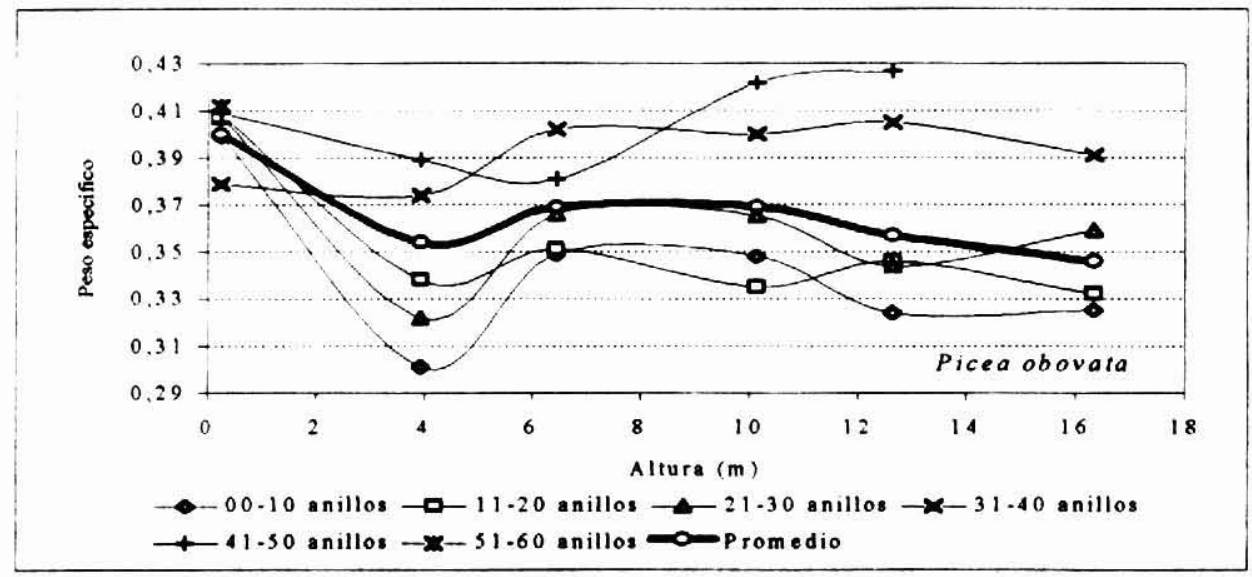

Figura $N^{\circ}$ 1. TENDENCIA DEL PESO ESPECIFICO PROMEDIO PONDERADO A VOLUMEN SATURADO DE LOS INCREMENTOS SEGÚN NÚMERO DE ANILLOS DESDE LA MÉDULA EN RELACIÓN A LA ALTURA DEL ÁRBOL EN IA MADERA DE Picea obovata Ledeb. 
Se puede observar que para cada altura existe un amplio rango de variación, siendo la excepción los valores de este atributo determinados para el menor nivel, los que oscilan entre 0,379 y 0,412 . Por otra parte, hacia la periferia del fuste ( 31 a 60 años) se presentan los máximos pesos específicos en relación con la altura del árbol.

Para Ciprés de Lawson no se encontró una tendencia definida en el comportamiento del peso especifico, caracterizándose más bien por las fuertes oscilaciones de los valores a lo largo del fuste (Cuadro $\mathrm{N}^{\circ} 4$ ). La Figura $\mathrm{N}^{\circ} 2$ ilustra el comportamiento de los valores en relación a la altura del árbol según sectores de edad equivalentes en madera de Chamaecyparis lawsoniana (A. Murray) Parlatore.

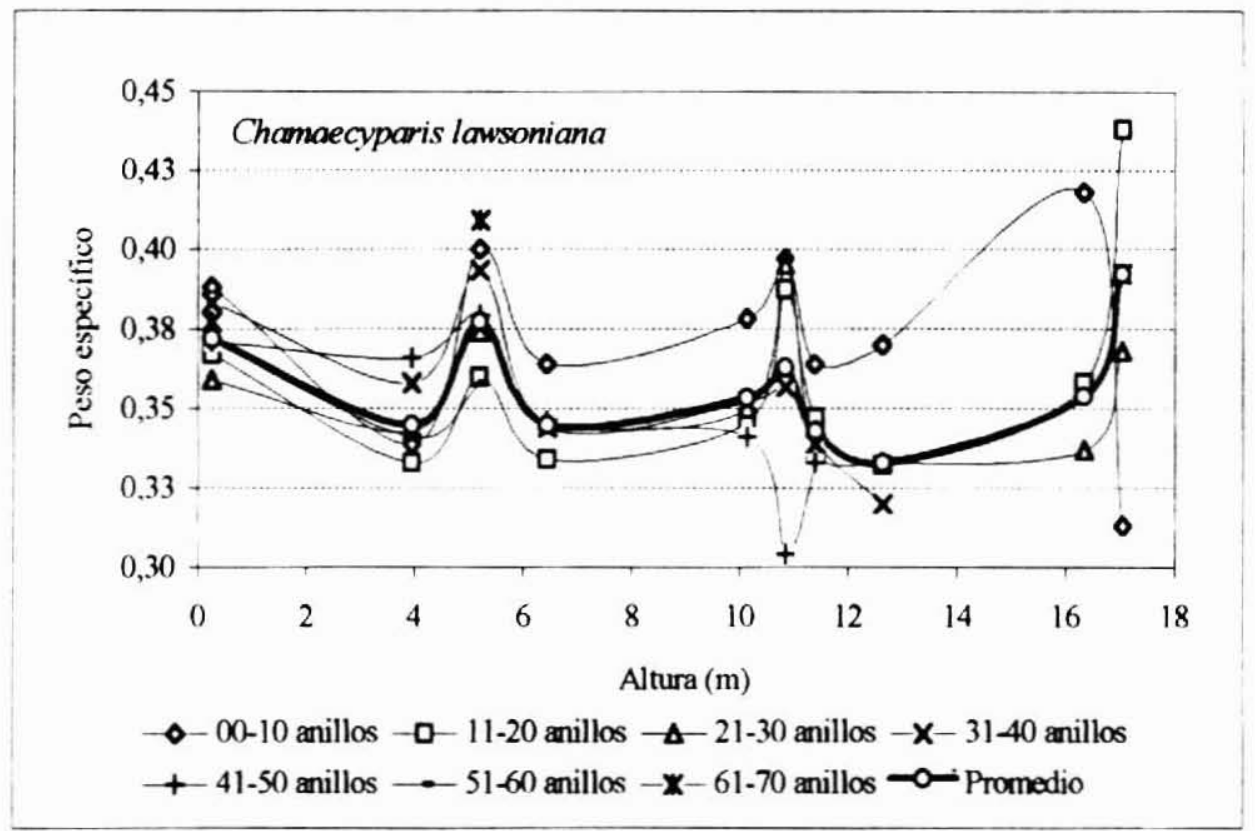

Figura $\mathrm{N}^{\circ}$ 2. TENDENCIA DEL PESO ESPECIFICO PROMEDIO PONDERADO A VOLUMEN SATURADO DE LOS INCREMENTOS SEGÚN NÚMERO DE ANILLOS DESDE LA MÉDULA EN RELACIÓN A LA ALTURA DEL ÁRBOL EN LA MADERA DE Chamaecyparis lawsoniana (A. Murray) Parlatore.

Para la mayor parte de las alturas los valores más elevados se presentan entre la médula y los 10 años de edad, comportamiento que sobre los $10,9 \mathrm{~m}$ se muestra también para los anillos 11 a 20. En relación a la dispersión de los valores en torno al promedio ponderado, en general. los distintos sectores intraincrementales siguen igual tendencia 
a lo largo del fuste. apreciándose además un incremento de los valores sobre los 11,40 m.

En el Cuadro $\mathrm{N}^{\circ} 4$ se entregan los valores para Pinus strobus $L$., los que muestran una tendencia al aumento a medida que se incrementa la altura sobre el tronco. En la Figura $\mathrm{N}^{0} 3$ se puede observar el comportamiento del peso especifico promedio ponderado según sectores intraincrementales equivalentes en relación a la altura del árbol en la especie Pino Blanco del Este.

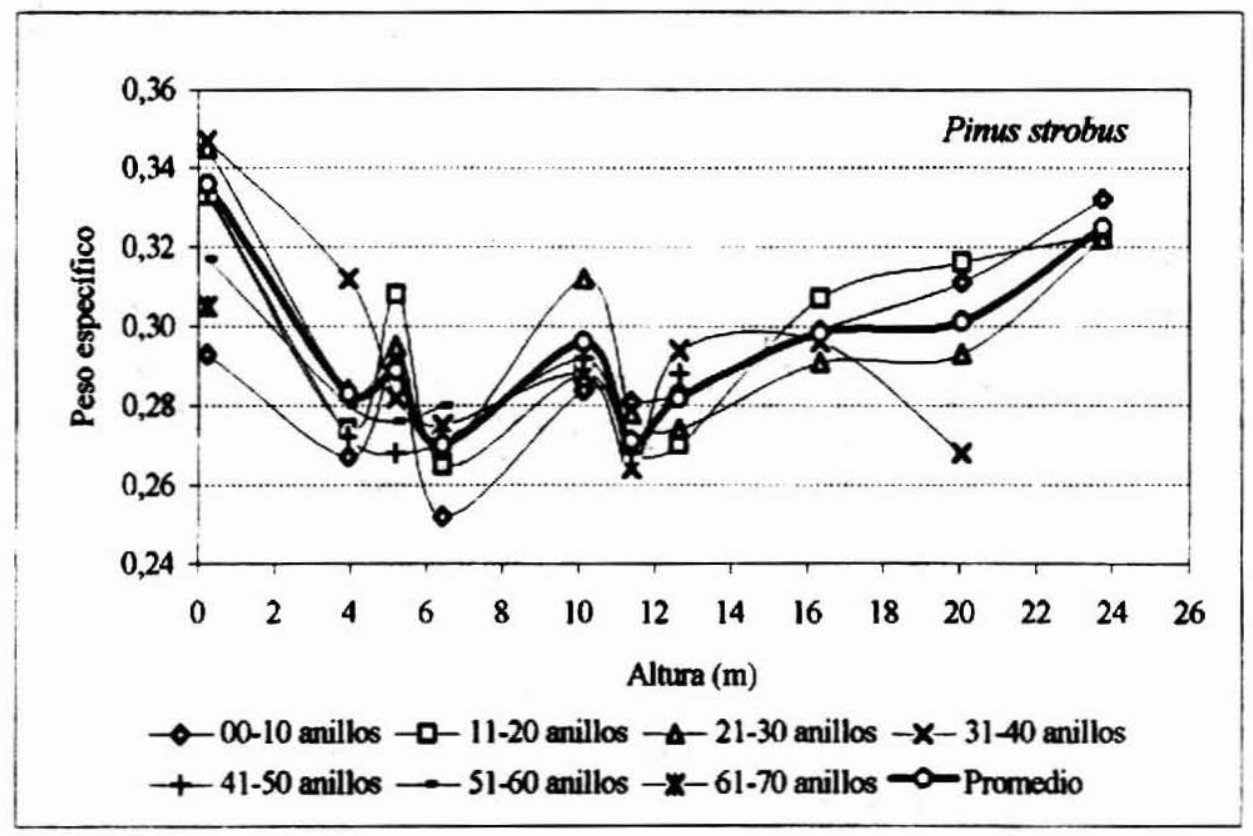

Figura $N^{\circ}$ 3. TENDENCTA DEL PESO ESPECIFICO PROMEDIO PONDERADO A VOLUMEN SATURADO DE LOS INCREMENTOS SEGÚN NÚMERO DE ANILLOS DESDE LA MÉDULA EN RELACIÓN A LA ALTURA DEL ÁRBOL EN LA MADERA DE Pinus strobus $L$.

La mayor variabilidad del peso especifico en torno al valor promedio ponderado se presenta para la altura de $0.25 \mathrm{~m}$. Sobre ésta, los valores se estabilizan y luego acusan un aumento moderado hacia la copa. 
Teadencia del Peso Eepecifico en Relación a la Edad Fisiológica del Árbol.

En el Cuadro $N^{\circ} 5$ se resumen los resultados obtenidos en las determinaciones de peso especifico ponderado en la dirección radial desde módula a corteza para las tres especies estudiadas.

\section{Cuadro $N^{0} 5$}

PBSO ESPECIFICO PROMEDIO PONDERADO A VOLUMEN SATURADO EN RELACTÓN A LA EDAD FISIOLOGICA DEL ÁRBOL (NÜMERO DE ANILLOS DESDE LA MÉDULA) SEGÚN ESPECIE

\begin{tabular}{|c|c|c|c|}
\hline $\begin{array}{c}\text { Número de anilloe } \\
\text { desde la módula }\end{array}$ & Peso Especifico (g/cm $\mathbf{c m}^{3}$ \\
\cline { 2 - 4 } & Picea obovata & $\begin{array}{c}\text { Chamaecyparis } \\
\text { lowsoniana }\end{array}$ & Pinus strobus \\
\hline $00-10$ & 0,348 & 0,383 & 0,286 \\
$11-20$ & 0,354 & 0,364 & 0,296 \\
$21-30$ & 0,363 & 0,357 & 0,297 \\
$31-40$ & 0,390 & 0,367 & 0,298 \\
$41-50$ & 0,409 & 0,354 & 0,290 \\
$51-60$ & 0,412 & 0,367 & 0,299 \\
$61-70$ & - & 0,382 & 0,305 \\
\hline
\end{tabular}

Las tendencias seguidas por los valores presentados se exponen en la Figura $\mathrm{N}^{\circ} 4$.

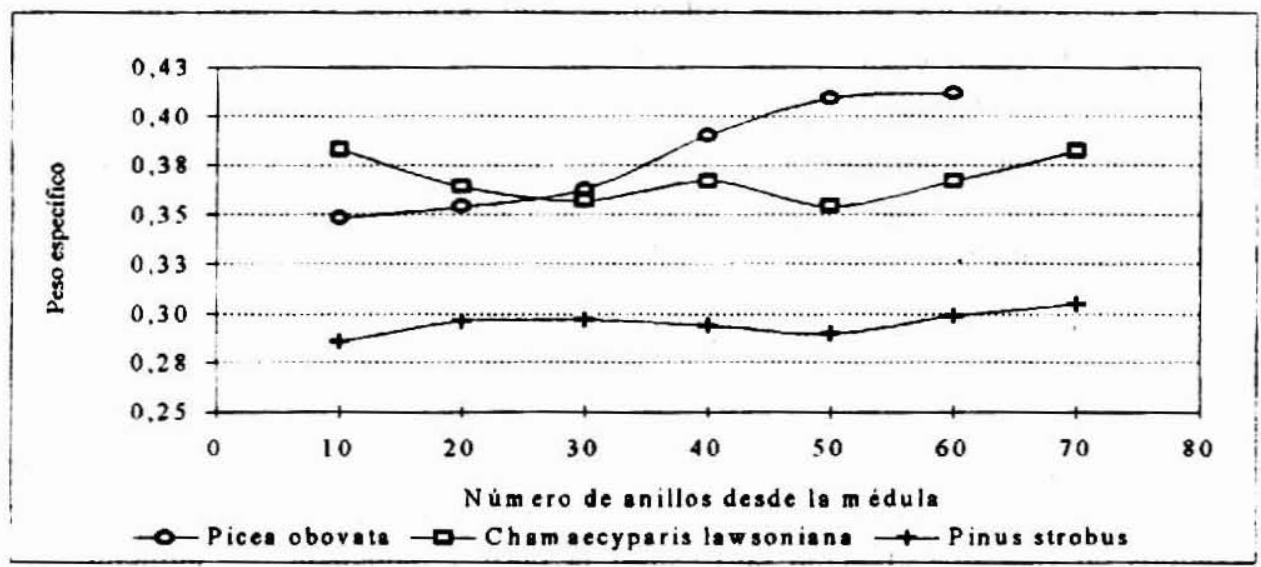

Figura $N^{\circ}$ 4. TENDENCIA DEL PESO ESPECTFICO PROMEDIO PONDERADO A VOLUMEN SATURADO DE LOS INCREMENTOS EN RELACIÓN AL NÚMERO DE ANILLOS DESDE LA MÉDULA SEGÚN ESPECIE. 
Para Picea Siberiana se observa un claro aumento del peso especifico promedio ponderado con la edad del árbol. En las especies Ciprés de Lawson y Pino Blanco del Este, en cambio, se presenta una tendencia constante con leve incremento desde la médula hacia las capas externas.

Tendencia de la Longitud de Traqueida en Relación a la Altura del Árbol.

A través de el Cuadro $\mathrm{N}^{\circ} 6$ podemos reconocer la tendencia seguida por los valores de longitud de traqueida hacia la copa del árbol.

\section{Cuadro $\mathrm{N}^{\circ} 6$}

PROMEDIO PONDERADO DE LONGITUD DE TRAQUEIDA EN RELACIÓN A LA ALTURA DEL ÁRBOL SEGÚN ESPECIE

\begin{tabular}{|c|c|c|c|}
\hline \multirow{2}{*}{ Altura } & \multicolumn{3}{|c|}{ Longitud de Traqueida (mm) } \\
\cline { 2 - 4 }$(\mathrm{m})$ & Picea obovata & $\begin{array}{c}\text { Chamaecyparis } \\
\text { lawsoniana }\end{array}$ & Pinus strobus \\
\hline 0.25 & 2,54 & 1,73 & 2,52 \\
10.15 & 2,80 & 2,65 & - \\
11,40 & - & - & 2,86 \\
16,35 & 2.56 & 2.44 & - \\
23,75 & - & - & 2,42 \\
\hline
\end{tabular}

De acuerdo con los resultados obtenidos. sólo podemos establecer que el largo celular aumenta desde la base del árbol hasta cierta altura en el fuste. disminuyendo en lo sucesivo de modo progresivo hacia las partes altas del mismo.

En relación al comportamiento a lo largo del fuste según sectores intraincrementales equivalentes para las tres especies la zona de madera juvenil entrega las menores longitudes en comparación a la madera adulta.

Tendencia de la Longitud de Traqueida en Relación a la Edad Fisiológica del Árbol.

En el Cuadro $N^{0} 7$ podemos reconocer la tendencia seguida por los valores promedios ponderados de longitud de traqueida hacia la periferia del fuste. Los valores recogidos en esta investigación corroboran la clásica tendencia: un aumento de la longitud celular a medida que nos alejamos del eje central del árbol, para luego alcanzar un valor constante o descender hacia la corteza. En efecto, para las tres coníferas analizadas se observa un incremento de la longitud celular con la edad fisiológica, tendiendo a la estabilización hacia la periferia del tronco. 


\section{BIBIDOTECA}

INSTITUIO FORESTAL

Cuadro No?

PROMEDIO PONDERADO DE LONGTTUD DETTAQUEIDA EN RELACIÓN A LA EDAD FISIOLOGICA DEL ARBOL (NUMERO DE ANILLOS DESDE LA MÉDULA) SEGÚN ESPECIE

\begin{tabular}{|c|c|c|c|}
\hline \multirow{2}{*}{$\begin{array}{c}\text { Nümero de anillos } \\
\text { desde la médula }\end{array}$} & \multicolumn{3}{|c|}{ Longitud de Traqueida (mm) } \\
\cline { 2 - 4 } & Picea obovata & $\begin{array}{c}\text { Chamaecyparis } \\
\text { lawsoniana }\end{array}$ & Pimus strobus \\
\hline $00-10$ & 2,00 & 1.46 & 1,97 \\
$11-20$ & 2.67 & 1.79 & 2.43 \\
$21-30$ & 3,00 & 2.12 & 2,83 \\
$31-40$ & 2,83 & 2,50 & 2.91 \\
$41-50$ & 2,53 & 2.45 & 2.94 \\
$51-60$ & 2,78 & 2,30 & 2,77 \\
\hline
\end{tabular}

De este modo, Picea Siberiana alcanza el máximo promedio ponderado entre los 21 y 30 años de edad ( $3.00 \mathrm{~mm})$; máximo que para Ciprés de Lawson se presenta entre los 31 y 40 años $(2.50 \mathrm{~mm})$ Con una estabilización de sus valores entre los anillos 21 y 50 . Pino Blanco del Este alcanza en esta última etapa su máximo valor $(2,94 \mathrm{~mm})$.

Analizando para cada especie el comportamiento de la longitud de traqueida en tres diferentes niveles de altura, se pudo apreciar que el incremento de la longitud desde la médula hacia la corteza se cumple en todos los casos: siendo la zona de tocón la que muestra las menores longitudes en relación a los dos restantes niveles de altura.

\section{Tendencia del Coarseness de Madera en Relación a la Altura del Árbol.}

A través del Cuadro $\mathrm{N}^{\circ} 8$ es posible reconocer la tendencia seguida por los valores de coarseness de madera hacia la copa del árbol.

Cuadro $\mathrm{N}^{\circ} 8$

PROMEDIO PONDERADO DE COARSENESS DE MADERA EN RELACION A LA ALTURA DEL ARBOL SEGÚN ESPFCIE

\begin{tabular}{|c|c|c|c|}
\hline \multirow{2}{*}{$\begin{array}{c}\text { Altura } \\
(\mathrm{m})\end{array}$} & \multicolumn{3}{|c|}{ Coarseness de Madera (dg) } \\
\cline { 2 - 4 } & Picea obovata & $\begin{array}{c}\text { Chamaecyparis } \\
\text { lawsoniana }\end{array}$ & Pinus strobus \\
\hline 0.25 & 16,35 & 13,78 & 20.60 \\
10,15 & 19,48 & 12,34 & - \\
11.40 & - & - & 19.10 \\
16,35 & 16,44 & 11.68 & - \\
23,75 & - & - & 16,47 \\
\hline
\end{tabular}


En Picea Siberiana, al igual que en el caso de la longitud de traqueida, sólo podemos deducir que el coarseness aumenta desde la base del árbol hasta cierta altura en el fuste, tras lo cual su magnitud disminuye hacia la copa. Para Ciprés de Lawson se detecta un descenso constante y progresivo del coarseness con el incremento de la altura en el árbol. La especie Pino Blanco del Este, finalmente, muestra los mayores valores de coarseness y, como lo expone el Cuadro $\mathrm{N}^{\circ} \mathbf{8}$, la tendencia es la de una disminución con el incremento en altura.

Por último, cabe hacer notar la similitud a variación entre los valores de longitud de traqueida y coarseness según altura para la especie Picea obovata Ledeb., semejanza que ya ha sido detectada en otras especies coníferas.

\section{Tendencia del Coarseness de Madera en Relación a la Edad Fisiológica del Árbol.}

En el Cuadro $\mathrm{N}^{\circ} 9$ podemos observar la tendencia seguida por los valores promedios ponderados de coarseness de madera hacia la periferia del fuste.

En términos generales, se presenta un aumento de los valores con la edad siendo más notable el fenómeno en el caso de Pino Blanco del Este, especie en la cual la tasa de incremento entre la zona medular ( 0 a 10 años) y la periferia (41 a 50 años) alcanza el $56,5 \%$.

\section{Cuadro $\mathrm{N}^{\circ} 9$}

PROMEDIO PONDERADO DE COARSENESS DE MADERA EN RELACIÓN A LA EDAD FISIOLÓGICA DEL ÁRBOL (NÚMERO DE ANILLOS DESDE LA MÉDULA) SEGÚN ESPECIE

\begin{tabular}{|c|c|c|c|}
\hline \multirow{2}{*}{$\begin{array}{c}\text { Número de } \\
\text { anillos desde la médula }\end{array}$} & \multicolumn{3}{|c|}{ Coarseness de la Madera (ng/100m) } \\
\cline { 2 - 4 } & Picea obovata & $\begin{array}{c}\text { Chamaecyparis } \\
\text { lawsoniana }\end{array}$ & Pinus strobus \\
\hline $00-10$ & 13,01 & 11.78 & 14.52 \\
$11-20$ & 16.96 & 13.19 & 18.73 \\
$21-30$ & 21,35 & 13.90 & 19.46 \\
$31-40$ & 18.65 & 12.71 & 21.44 \\
$41-50$ & 16.82 & 15.27 & 22.72 \\
$51-60$ & 18.44 & 14.38 & 21.30 \\
\hline
\end{tabular}

Ciprés de Lawson -que presenta cierta estabilidad con una tendencia al incremento de los valores- muestra, sin embargo, los menores indices del atributo evaluado, situación semejante a la detectada para la variación en altura. Picea Siberiana, por otro lado, tiende a incrementar los valores hasta cierta edad en el árbol ( 0 y 30 anillos) para después disminuir hacia la periferia del fuste, con un incremento final hacia el último sector anular. 
Por último, cabe destacar la notable similitud en las tendencias seguidas para los valores de coarseness y de longitud de traqueida según edad en Picea obovata Ledeb..

\section{CONCLUSIONES}

Los valores promedios ponderados de peso específico a volumen saturado, longitud de traqueida y coarseness de madera se encuentran dentro del margen general establecido por la literatura para especies madereras (coniferas).

El peso específico depende de la posición en altura en el fuste: disminuye hacia la copa en la especie Picea obovata Ledeb. y aumenta en dicha dirección en el caso de Pinus strobus L. En Chamaecyparis lawsoniana (A. Murray) Parlatore, en tanto, no se apreció una tendencia definida en el comportamiento de esta variable.

El peso especifico en Picea obovata Ledeb. varía en función directa con respecto a la edad del árbol (número de anillos desde la médula). Chamaecyparis lawsoniana (A. Murray) Parlatore y Pinus strobus L., presentaron, en cambio, una tendencia relativamente constante en el comportamiento de este atributo.

La longitud de traqueida en las especies evaluadas aumenta desde la base del árbol hasta cierta altura en el fuste. disminuyendo progresivamente hacia las partes altas del mismo.

En cada una de las especies estudiadas, la longitud de traqueida incrementa con tendencia a la estabilización hacia la periferia del tronco.

En Picea obovata Ledeb. el coarseness aumenta desde la base del árbol hasta cierta altura en el fuste. tras lo cual su magnitud decrece hacia la copa. En Chamaecyparis lawsoniana (A. Murray) Parlatore y Pinus strobus L.. en tanto, éste desciende con el incremento de la altura en el árbol.

El coarseness aumenta con el número de anillos desde la médula en las tres coniferas evaluadas.

Se observó una notable similitud en el comportamiento adoptado por los valores de longitud de traqueida y coarseness de madera, tanto en altura como en edad. en la especie Picea obovata Ledeb..

\section{REFERENCIAS}

Britt, K. 1966. Fiber Coarseness in Wood. Tappi, 49(5): 202-6.

Casey, J., 1960. Papermaking. Vol. 2. Pulp and Paper Chemistry and Chemical Technology, Second Edition. Interscience Publishers, Inc. New York. USA.

CONAF, 1975. Plan de Manejo para la Reserva Forestal de Malleco. Dirección Regional, Oficina de Programación XI Zona CONAF. IX Region. 
CONAF, 1991. Bases Licitación "Estudios Básicos sobre Volumetria, Crecimiento y Taxonomia en Rodales de Coníferas Exóticas en Sector Los Guindos-Reserva Forestal de Malleco". Dirección Regional, DX Región.

Daniel, P., Helms, V. y Baker, F., 1982. Principios de Silvicultura. Traducido de la Segunda Edición Inglesa por Ramón Elizondo Mata. Mc Graw-Hill. México, D.F.

Delmastro, R., Diaz-Vaz, J. y Schlatter, E., 1980. Variabilidad de las Características Tecnológicas Hereditarias del Pinus radiata D. Don. Revisión Bibliográfica. Proyecto CONAF/PNUD/FAO/CHI/76/003C, Documento de Trabajo $\mathrm{N}^{\mathrm{O}} 34$.

Einspahr, D.W., 1964. Correlations Between Fiber Dimensions and Fiber and Handsheet Strength Properties. Tappi, 47(4): 180-3.

González, J. y Molina, J., 1989. Consideraciones sobre los tratamientos silviculturales y los rendimientos cuantitativos y cualitativos en madera pulpable de Pino Radiata. Documento Técnico $\mathrm{N}^{\mathrm{O}} 38$, Revista Chile Forestal. Corporación Nacional Forestal, Santiago de Chile.

Gysling. J., 1982. Influencia del Peso Especifico en la Rotación Económica de plantaciones de Pinus radiata $D$. Don para producción de Pasta y Papel. Tesis de Ing. For. Universidad de Chile, Escuela de Ciencias Forestales. Santiago de Chile.

Laboratorio de Productos Forestales (L P. F.), 1985. Factores que influyen en la calidad de la madera y que afectan la calidad de la pulpa. Dirección de Investigaciones Tecnológicas y Asistencia Técnica. Universidad de Concepción, Chile

Martínez, F. y Pacheco, L, 1990. Una visión de la pulpa kraft de Pino Radiata Chileno. Caracterización, Ventajas comparativas y Potencialidades. Asociación Técnica de Celulosa y Papel (ATCP). Revista Celulosa y Papel 6(3): 6-14. Chile.

Technical Association of the Pulp and Paper Industry (TAPPI), 1989. "Tappi Test Methods". Atlanta, USA.

Vargas, G., 1991. Peso Específico de la Madera de Compresión en Pino Insigne (Pinus radiata D. Don) crecido en la Décima Región. Tesis de Ing. For. Universidad Austral de Chile, Fac. de Ciencias Forestales.

Zobel, B..J y Talbert, J., 1988. Técnicas de Mejoramiento Genético en árboles Forestales. Versión Española: Manuel Guzmán Ortíz. Editorial Limusa, México, D.F.. 